\title{
Probabilistic assessment of pile capacity based on CPTu probing including random pile foundation depth
}

\author{
Marek Wyjadłowski ${ }^{1, *}$, Irena Bagińska ${ }^{1}$, and Jakub Reiner $^{1}$ \\ ${ }^{1}$ Wroclaw University of Science and Technology, Faculty of Civil Engineering, Wroclaw, Poland
}

\begin{abstract}
The modern recognition of subsoil with the use of CPTu static probes allows to obtain detailed information necessary for the designing. Registered basic two quantities, i.e. cone resistance $q c$ and friction on the sleeve $f s$, often become direct data, which allow to estimate bearing capacity of the base and the side surface of the pile. Direct methods use similarity of the pile work and piezo-cone work during the examination. An important design stage is the appropriate development of measurement data prior to the commencement of the procedure of determining the pile bearing capacity. Algorithms generated on the basis of empirical experiments are often applied with the simultaneous use of test loads. The probabilistic approach is also significant, because it enables objective assessment of the reliability level of performed design calculations. This work contains an analysis of the impact on the estimated bearing capacity and reliability of a pile of variable random depth of the pile base. It also includes the determination of probabilities of obtaining the assumed safety index for the designed solution at random foundation depth.
\end{abstract}

\section{Introduction}

The CPT test specified in standard [1] refers to mechanical and electrical cone penetration tests. The assessment of pile capacity employing the results of CPT surveys provides the most satisfactory results for the following reasons:

- the shape of the penetrometer reproduces the shape of the pile

- the state of soil during the test is comparable to that in which the pile will be executed,

- the process of the CPT test is quasi-static,

- during penetration, the probe generates a limit state in the surrounding soil, both under the cone and along the friction sleeve.

The majority of pile design methods using the results of CPT test are based on employing a static formula for ultimate bearing capacity of a pile. In compliance with Eurocode 7 [2] recommendations, pile foundation design is based on static load test results, and additionally also on other empirical and analytical methods.

During the design of piles, a safety index is usually assumed as a safety measure, which describes the ratio between the pile bearing capacity and the load that has an impact on it.

\footnotetext{
Corresponding author: marek.wyjadlowski@pwr.edu.pl
} 


$$
F=\frac{R}{N}
$$

where:

$R$ - limit bearing capacity of the pile;

$N$ - acting design load;

$F$ - safety index (partial coefficient for bearing capacity $\gamma t$ according to Eurocode $7[2,3]$ ). However, the provided index measure is not always sufficient. Due to the high uncertainty of geotechnical recognition, which results among others from the long-term nature and complexity of geological processes, as well as the limited quantitative possibilities of subsoil examination, the ground should be treated as a random centre. While also taking into account the fact associated with performance errors, which are also random, the safety assessment on the basis of a single parameter may not be sufficient and it should be supplemented by a probabilistic measure. This measure may be the probability of a failure occurrence that takes into account the randomness of occurrence of individual factors $[4,5,6] 7,8]$. Assuming that the safe safety index amounts to 1.4 , while the lack of its achievement means a failure, this probability may be described with the formula (2):

$$
p_{f}=P[F \leq 1.4]=\int_{-\infty}^{1.4} f(F) d F
$$

where:

$p_{f}$ - probability of failure

$\mathrm{P}[F \leq 1.4]$ - probability of such event that the safety index is lower than 1.4 ;

$f(F)$ - density function of the probability of failure.

Safety index value for the pile was adopted at the lvel of 1.4 in accordance with the

recommendations of Eurocode 7.

\section{Pile Bearing Capacity Estimation}

In the scope of vast majority of methods that allow to estimate the pile bearing capacity on the basis of results static probing results, the bearing capacity of a pile can be calculated from formula (3):

$$
R_{c k}=R_{b, k}+R_{s, k}=A_{b} \cdot q_{b, k}+\sum_{i} A_{s i} \cdot q_{s, k, i}
$$

$R_{b, k}$-ultimate soil resistance under pile base [kN],

$R_{s, k}$ - ultimate soil resistance on pile side [kN],

$A_{b}$ - surface area of the pile base $\left[\mathrm{m}^{2}\right]$

$A_{s i}$ - surface area of pile side within the $\mathrm{i}^{\text {th }}$ layer of soil $\left[\mathrm{m}^{2}\right]$,

$q_{b, k}-$ unit ultimate resistance under pile base $[\mathrm{kPa}]$,

$q_{s, k, i}$ - unit ultimate resistance on pile side within the $\mathrm{i}^{\text {th }}$ layer of soil [kPa].

The values $q_{b, k}$ and $q_{s, k, i}$ are calculated from the following formulas:

$$
\begin{gathered}
q_{b, k}=\psi_{1} \cdot \underline{q}_{c} \\
q_{s, k, i}=\frac{\underline{q}_{c s i}}{\psi_{2}} \quad \text { or } q_{s, k, i}=\frac{f_{s i}}{\psi_{3}}
\end{gathered}
$$

where:

$\underline{q}_{c}$ - averaged unit soil resistance under probe cone near the pile base $[\mathrm{kPa}]$, $\underline{q}_{c s i}$ - averaged unit soil resistance under probe cone within the $\mathrm{i}^{\text {th }}$ layer $[\mathrm{kPa}]$, 
$f_{s i}-$ averaged unit soil resistance on the side of the probe sleeve within the $\mathrm{i}^{\text {th }}$ computational layer $[\mathrm{kPa}]$,

$\psi_{1}$ - bearing capacity coefficient of pile base,

$\psi_{2}$ - bearing capacity coefficient of pile side within the $\mathrm{i}^{\text {th }}$ computational layer (allowing for resistance $q_{c}$ ),

$\psi_{3}$ - bearing capacity coefficient of pile side within the $\mathrm{i}^{\text {th }}$ computational layer (allowing for resistance $f_{s}$ ).

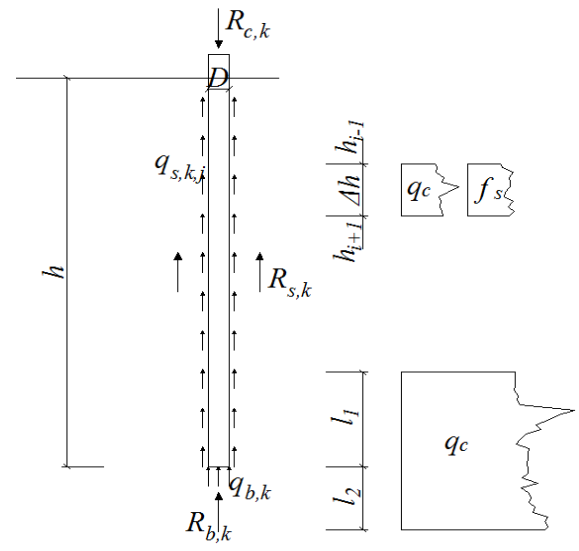

Fig. 1. Scheme of calculating pile bearing capacity.

Computational methods vary according to the method of determining the values $\underline{q}_{c}, \underline{q_{c s i}}$, $f_{s i}$ and adopting the values of coefficients $\psi_{i}$, which depend on the technology of pile execution and the type and state of the soil in the immediate vicinity of the pile.

Determining the values $\underline{q}_{c}, \underline{q}_{c s i}$ and $\underline{f}_{s i}$ can be described by these general formulas:

$$
\begin{array}{r}
\underline{q}_{c}=\frac{1}{l_{1}+l_{2}} \int_{h-l_{1}}^{h+l_{2}} q_{c}(h) d h \\
\underline{q_{c s i}=}=\frac{1}{\Delta h} \int_{h_{i}-1}^{h_{i}} q_{c}(h) d h \\
f_{s i}=\frac{1}{\Delta h} \int_{h_{i}-1}^{h_{i}} f_{s}(h) d h
\end{array}
$$

$l_{l}, l_{2}$ - the range of averaging computational layers above and below the level of the designed pile base,

$\Delta h$ - thickness of the computational layer of soil adopted for calculations.

Fundamentally, all methods for estimating the pile bearing capacity on the basis of CPTu results are based on equation (3). The differences consists of assuming in the calculations different empirical values of the following coefficients: $\psi_{1}, \psi_{2}$ and $\psi_{3}$, which depend among others on the type of ground and technology of pile manufacture. Moreover, the big difference is the selection of different thicknesses of the $l_{1}$ and $l_{2}$ areas, which have a direct impact on the obtained averaged unit ground resistance for the base and the side surface of the pile from the measured values $q_{c}$ and $f_{s}(6,7,8)$.

\section{Estimation of the pile bearing capacity according to EC7}

The calculation method is broadly consistent with formula (3). The parameters necessary for calculations are shown in the Figure 4. In the case of this method, there's are no deterministically given range guidelines $l 1$ and $l 2$. These areas are only suggested as 
$l_{1}=8 D e q$, where $D e q$ is the pile replacement diameter and $l_{2}=d_{c r i t}$, where $d_{c r i t}$ is the depth in area below the pile base, in the range between $0,8 D e q$ and $4 D e q$, where pcImean $\left(q_{c}\right)$ achieves the minimum value.

\section{Estimation of the pile bearing capacity}

\subsection{Geotechnical Site Conditions}

Geotechnical investigation was conducted in the area of Wrocław/Poland by CPTu probe drillings and laboratory tests. Uncontrolled embankment was located in the subsoil to a depth of approx. $3.5 \mathrm{~m}$. Then, there's a layer of cohesive soils (silty clays) with a thickness of $1 \mathrm{~m}$. Below the clays, only the non-cohesive soils occur until the very end of registration, in which the largest thickness constitute silty sands in the medium-compacted and compacted state. The ground water table is tight and it is located just below the layer of clays. The course and arrangement of layers are well illustrated by cited measurement data from the $\mathrm{CPTu}$ probing, which are presented in Figure 2.

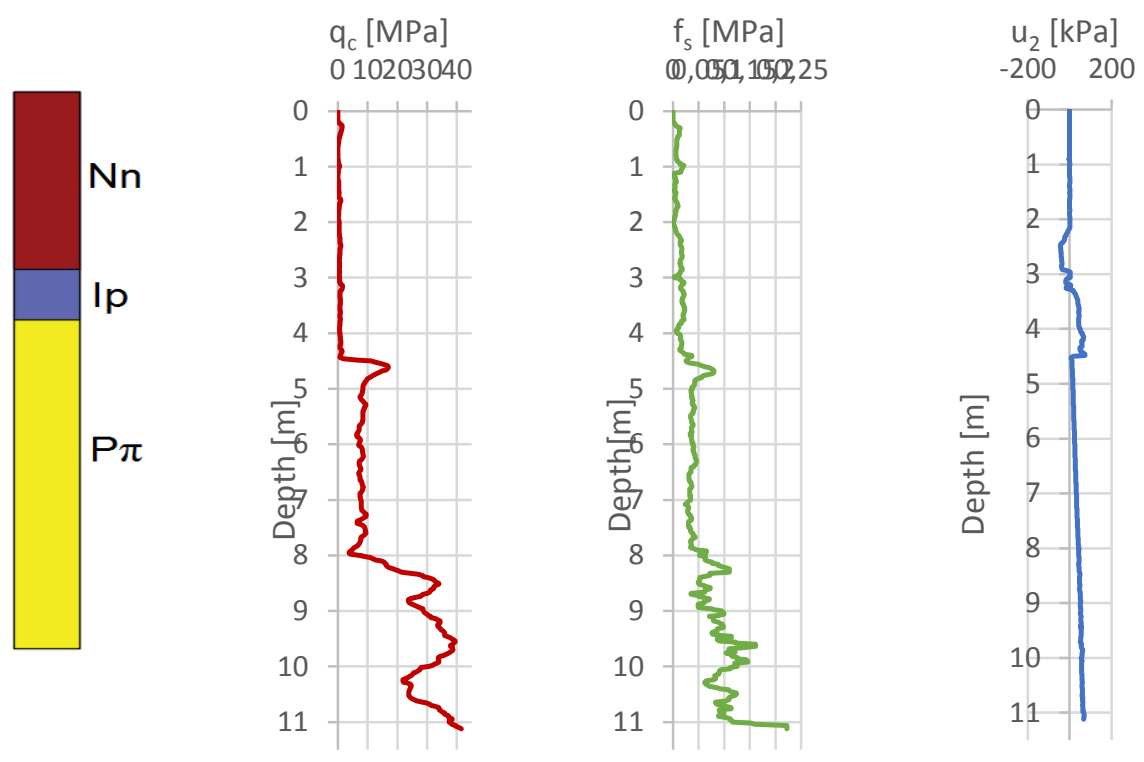

Fig. 2. The measurement data of CPTu.

Due to the existing ground conditions and load value, an intermediate foundation of the building was planned through CFA reinforced concrete columns carried out with the diameter of $430 \mathrm{~mm}$. Registrations of the cone resistance $q c$ and resistance on the friction sleeve $f_{S}$ were directly implemented to algorithms allowing to estimate the pile bearing capacity with the cited methods.

Characteristic bearing capacity required for the design for pressing of a single reinforced concrete column amounted to $1216 \mathrm{kN}$, while the calculated one by $40 \%$ more, i.e. $1702.4 \mathrm{kN}$. The end of the column was planned at a depth of $9.2 \mathrm{~m}$. below ground level, i.e. in the layer of silty sand, in a very compacted state with $\mathrm{I}_{\mathrm{D}} \geq 0.80$. After performance of the foundation, the pile that was the closest to the previously conducted CPT probing was 
subjected to a test load. The obtained results became the basis for verification of the performed calculations and analysis of the obtained results.

\subsection{Deterministic assessment of the pile bearing capacity}

It was assumed that the geotechnical recognition provides data for the design of deterministic nature. This analysis was conducted by comparing the limit bearing capacity value obtained from the static load test and the pile bearing capacity, determined with the use of [3] method.

Principles of carrying out load test and estimating the bearing capacity of the pile base are described in detail in $[6,7,8]$.

According to the detailed design, it was assumed that depth of the pile foundation amounted to $9.2 \mathrm{~m}$. below ground level, while the pile diameter amounted to $430 \mathrm{~mm}$.

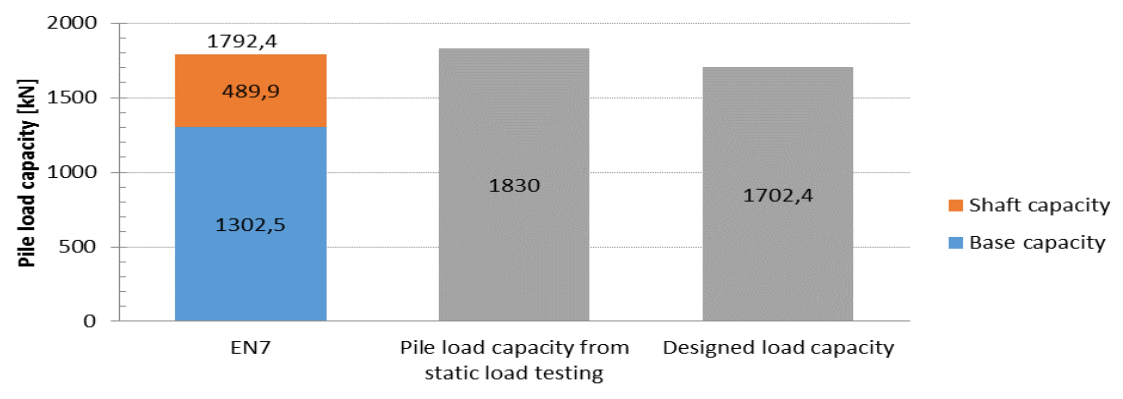

Fig. 3. Comparative graph of obtained pile bearing capacity.

The bearing capacity calculated in accordance with the guidelines of EN7 amounts to $1792.4 \mathrm{kN}$, while the estimation accuracy is lower than $2.5 \%$. The pile bearing capacity for deterministic calculations is shown in the Figure 3.

\subsection{Probabilistic assessment of the pile bearing capacity}

In the presented task, the random variable is the difference of pile base ordinate in relation to the designed ordinate. Reliability problems were defined in many previous studies, i.e., [9], Therefore, the probabilistic measure will be the probability of a failure occurrence, in the form of insufficient pile bearing capacity due to the random depth of its foundation. Failure is defined as a situation, in which the pile foundation on other depth than the designed depth will result in achieving a safety index lower than 1.4 [2].

Based on the contractors' experience, it was assumed that the probability of foundation performance at the given depth has a normal distribution (9), while the expected value of foundation depth will be greater than the planned foundation depth by $0.2 \mathrm{~m}$ and it will amount to $9.4 \mathrm{~m}$. The standard deviation was assumed as $0.2 \mathrm{~m}$ (Figure 4 ).

$$
f_{H}(H)=\frac{1}{\sigma_{H} \sqrt{2 \pi}} e^{-\frac{1}{2}\left(\frac{H-m_{H}}{\sigma_{H}}\right)^{2}}
$$

where:

$m_{H}-$ expected value $[\mathrm{m}] ; \quad \sigma_{H}-$ standard deviation $[\mathrm{m}]$. 


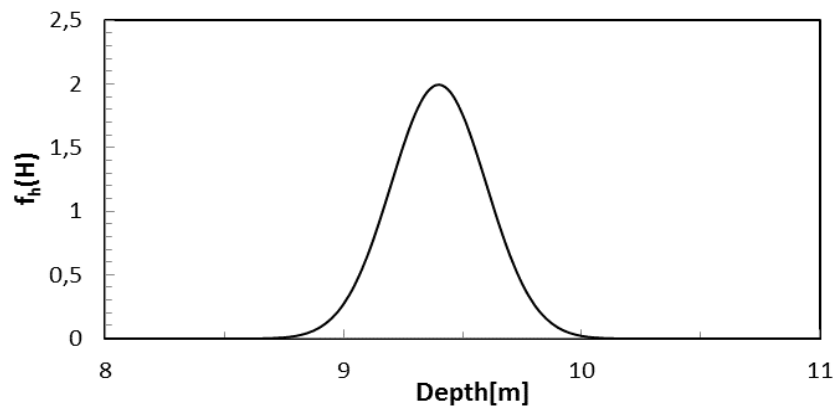

Fig. 4. Density function of the probability of variable random foundation depth according to eq. 9 .

In order to determine the probability of a failure, it's necessary to determine the relation between the bearing capacity and the depth of pile foundation. In the considered case, the estimated pile bearing capacity increases along with the foundation depth. In the method based on the guidelines of EN7, this increase is linear.

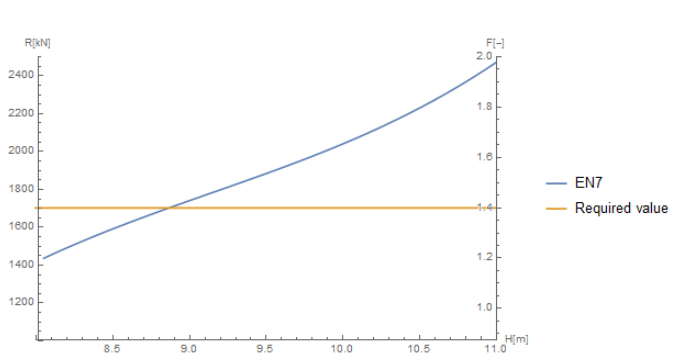

a)

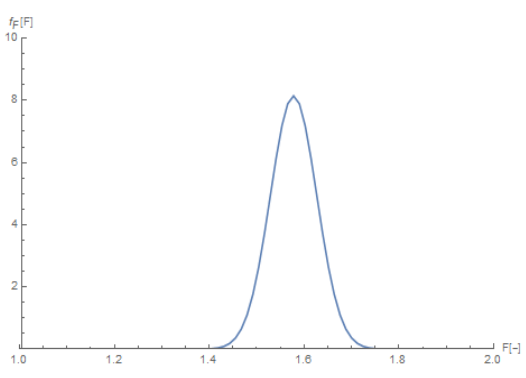

b)

Fig. 5. a) Safety index in the function of pile base ordinate b) Density function of the probability of safety index.

Considering the fact that the depth of pile performance is a random function, the function describing the variability of safety index depending on the foundation depth also becomes random.Using the transformational formula (10), it's possible do determine the density function of the probability of safety index on the basis of density function of the probability of variable random foundation depth and function describing relation between the safety index and the foundation depth. In the considered case, the relation (10) is important, because it was assumed that only one factor in the form of pile foundation depth has impact on the value of variable safety index [13].

$$
f_{F}(F)=\left\lceil\frac{d g^{-1}(F)}{d F}\right\rceil f_{h}\left[g^{-1}(F)\right]
$$

where :

$f_{F}(F)$ - density function of the probability of safety index;

$f h(H)$ - density function of the probability of pile foundation depth;

$H=g^{-1}(F)$ - reverse function recording the relation between safety index and pile foundation depth.

Similarly as the variability of pile bearing capacity was presented in the function of foundation depth, it is also possible to show the variability of safety index value $F$, along with the pile foundation depth, Figure 5a. 
Fig. $5 \mathrm{~b}$ shows the curve of density functions of the probability of safety indexes for the analyzed method of bearing capacity estimation, with the assumed distribution of foundation depth variability. Shape of the density function is similar to the assumed normal distribution of random variability of foundation depth (Figure 4), but it gains individual character of the curve due to the used transformation (10). In the considered depth range, the relation between the safety index and the foundation depth is similar to the nature of growth with depth.

In accordance with the guidelines of the EN0 standard [10], the designed object is classified as RC2 reliability class and CC2 consequence class. Such objects are characterized by an average threat to human life and significant consequences of a possible failure. In order for the structure to meet requirements of the standard, it is necessary to ensure design safety at the level of $p_{f} \sim 1 \cdot 10^{-4}$. For the assessment of design safety level, it's necessary to determine the probability of failure occurrence, which may be directly determined by using the graph of distribution function of the safety index. For the selected method of bearing capacity estimation, it's the point at which the distribution function intersects with the vertical axis, placed at the point with the value for which the probability of failure occurrence is checked. In our case, it's according to the guidelines [2] - the value of safety index equal to 1.4 (Figure 6).

Fig. 6. Distribution function of the safety index

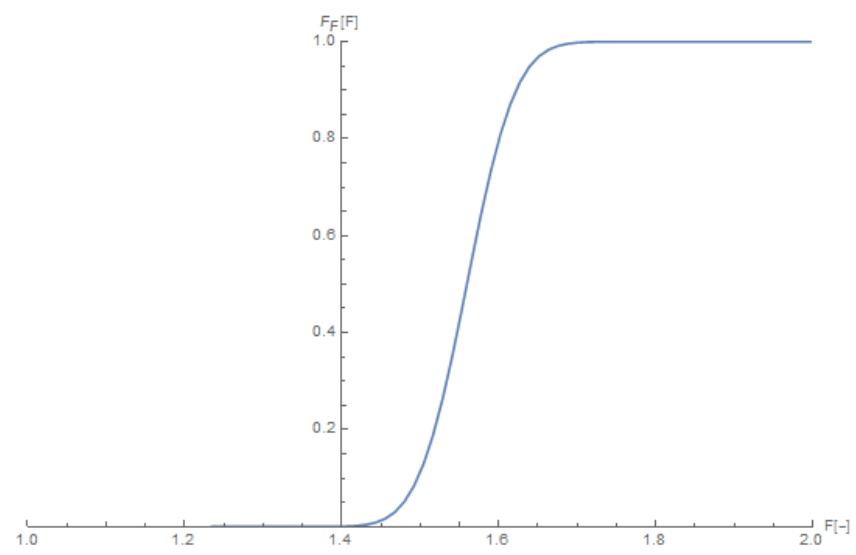

For the method based on the guidelines of Eurocode $7 p f=[F \leq 1,4]=1 \cdot 10^{-4}$, it is the probability level acceptable as safe for the RC2 reliability class and the $\mathrm{CC} 2$ consequence class.

\section{Summary}

The method based on the guidelines of [2], after taking into account the random depth of the pile foundation, ensures appropriate level of pile reliability. It meets the requirements for probabilistic measure, as well as deterministic measure.

It's necessary to emphasize that the obtained conclusions are binding only for the analyzed design situation and other geotechnical cases must be considered individually. This work verified the calculations of pile bearing capacity, determined on the basis of CPTu probing, with the results of test loads. The impact of foundation depth on the estimated bearing capacities of the pressed pile was also analyzed. The attention was drawn to the reliability of obtaining the assumed safety index for the performed estimations of bearing capacity, in the case of finishing the pile at a different depth than the designed depth. 


\section{References}

1. ISO 22476-1 Geotechnical investigation and testing - Field testing Part 1: Electrical cone and piezocone penetration test, 2012

2. EN 1997-10:2009 Eurocode 7: Geotechnical design - Part 1 : General Rules

3. EN 1997-2 : 2009 Eurocode 7 : Geotechnical design - Part 2: Ground investigation and testing

4. J.P. Li, J. Zhang, S.N. Liu, C.H. Juang, Soils and Found, 55, 637-649 (2015)

5. J. Lins da Silva, N. Aoki, Y.B.Franco, Dyna, 200, 247-252 (2017)

6. J. Rybak, IOP Conference Series - Materials Science and Engineering, 245, 1-8 (2017)

7. M. Baca, J. Rybak, A. Tamrazyan, T. Żyrek, 16th International Multidisciplinary Scientific GeoConference, 1, 945-950 (2016)

8. Z. Muszyński, J. Rybak, 14th International Multidisciplinary Scientific GeoConference, 2, 451-458 (2014)

9. M. Kawa, W. Puła, M. Suska, Studia Geotechnica et Mechanica, 38, 3-13 (2016)

10. EN 1990: 2004 Eurocode - Basis of structural design 